\title{
Methods and tools for geographical mapping and analysis in primary health care
}

\author{
Edgar Samarasundera ${ }^{1}$, Thomas Walsh ${ }^{2}$, Tao Cheng ${ }^{3}$, Ariane Koenig ${ }^{2}$, Kavita Jattansingh ${ }^{2}$, Alex Dawe ${ }^{2}$ \\ and Michael Soljak ${ }^{4}$ \\ ${ }^{1}$ Honorary Research Officer, Department of Primary Care and Public Health, Imperial College London, London, UK \\ ${ }^{2}$ Postgraduate Student, Department of Civil, Environmental and Geomatic Engineering, University College London, \\ London, UK \\ ${ }^{3}$ Senior Lecturer in Geoinformatics, Department of Civil, Environmental and Geomatic Engineering, University \\ College London, London, UK \\ ${ }^{4}$ Postgraduate Researcher, Department of Primary Care and Public Health, Imperial College London, London, UK
}

\begin{abstract}
Aim: The purpose of this paper is to review methods and tools for mapping, visualising and exploring geographic information to aid in primary health care (PHC) research and development. Background: Mapping and spatial analysis of indicators of locality health profiles and healthcare needs assessment are well-established facets of health services research and development. However, while there are a range of different methods and tools used for these purposes, non-specialists responsible for managing the use of such information systems may find knowing where to start and what can be done a relatively steep learning curve. In this review, health and sociodemographic datasets are used to illustrate some key methods, tools and organisational issues, and builds upon two recent reviews in this journal, respectively, focusing on geographic data sources and geographic concepts. Those familiar with mapping and spatial analysis should find this a useful review of current matters. Method: A thematic review is presented with illustrative case studies relevant to PHC. It begins with a section on visualising and interpreting geographic information. This is followed by a section critiquing analytical methods. Consideration is given to software and deployment issues in a third section. Content is based on domain knowledge of the authors as a team of geographic information scientists and a public health practitioner working in tandem, with its scope restricted to routine applications of mapping and analysis. Advanced techniques such as spatio-temporal modelling are not considered, neither are methodological technicalities, although guidance on further reading is provided. Summary: Geographical perspectives are now playing a significant role in PHC delivery, and for those engaged in informatics and/or managing population-level care, understanding key geographic information systems methods and terminologies are important as is gaining greater familiarity with institutional aspects of implementation.
\end{abstract}

Key words: inequalities; informatics; populations; socio-economic; statistics

Received 23 May 2011; accepted 7 August 2011; first published online 25 October 2011

\section{Introduction}

Geographical mapping and analysis of health and healthcare are well-established facets of

Correspondence to: Edgar Samarasundera, Department of Primary Care and Public Health, Imperial College London, Reynolds Building, St. Dunstan's Road, London W6 8RP, UK. Email: e.samarasundera@imperial.ac.uk

(C) Cambridge University Press 2011 epidemiological and health services analyses (Joseph and Phillips, 1984; Meade and Earickson, 2005: 381-400), with many studies specifically examining primary health care (PHC; eg, Guagliardo, 2004; Luo, 2004; Wang and Luo, 2005; Ricketts and Holmes, 2007; Busato and Kunzi, 2008). Much of the mainstreaming of health geography has been due to advances in geographic information systems (GIS) methods and technologies, 
and an associated range of geographically referenced (georeferenced) health and sociodemographic datasets (Samarasundera et al., 2010a). These trends have also resulted in the growth of interactive, internet-based health and healthcare atlases, as well as software tailored to health services applications, making mapping available to a wider healthcare audience. Examples include the World Health Organization (WHO) mapping and GIS programme (http://www.who.int/health mapping/en/), the North West England public health observatory mapping facility (http://www. nwpho.org.uk/healthgis/) in the United Kingdom and the New Zealand Ministry of Health intelligence tool (http://www.phionline.moh.govt.nz/).

The increasing application of GIS in PHC is part of this trend. However, making effective use of georeferenced datasets (Samarasundera et al., 2010a) in GIS software and e-health atlases requires an understanding of geographic concepts (Crooks and Andrews, 2009) as well as a managerial understanding of the purposes, scope and limitations of mapping and analysis methods. This review examines spatial mapping and analysis methods and tools for population-level management of PHC and health intelligence purposes, and also discusses the key institutional and implementation issues relevant to effective deployment of such methods and technologies.

\section{Mapping health and health care}

Maps are an effective form of visualising information on many topics pivotal to PHC research and development including identifying healthcare access disparities and visualising disease patterns (Walter, 1992; Lawson and Williams, 2001; Cossman et al., 2003). Nevertheless, maps require accurate interpretation and hence understanding both how to design maps effectively and interpret cartographic data are fundamental (Mackie and Sim, 2008). For example readers might 'see' morbidity patterns that do not actually exist due to the effects of the choice of colour scheme and data classes employed (Lawson, 2001).

\section{Colour schemes}

A fundamental aspect of visualising cartographic information is the use of colour schemes. Greyscale maps are very popular in academic journals, largely due to cost factors, but are effective in communicating quantitative information, with near-white colours typically representing low levels, and dark grey or black signifying high levels, of a given phenomenon such as disease prevalence. It should be noted that it is considered best practice among many cartographers not to use white to represent low levels, with white itself being reserved to indicate areas for which there are no data. Non-greyscale colour maps of course are also valuable, with single colour gradations and diverging schemes being two standard alternatives for representing numerical gradients. The former is essentially the same as greyscale but uses another colour instead, for example very light pink to very dark red.

Divergent schemes are also common but require more consideration. While map users frequently find the use of a two-colour divergent scheme useful for highlighting very high levels of a phenomenon, it has been found in many cartographic studies that some colour schemes aid visual interpretation while others typically cause confusion. For example there is support for the contention that red-blue colour schemes are generally preferable for disease map construction (eg, Walter and Birnie, 1991; Brewer and Pickle, 2002), other studies having found different schema to be effective, purple-green for example (Brewer et al., 1997). A divergent colour scheme that should be discouraged is red-green due to the interpretation difficulties it poses to those with partial colour loss.

\section{Data classification}

How data are classified into classes in map legends has long been a key aspect of cartographic design; this is because the choice of class interval types can alter a map user's perceptions of geographic information. There are many alternative classification systems available; common ones are equal interval categories, natural breaks in data, quantiles (eg, quartiles, quintiles, deciles) and classes based on standard deviations. The map producer is thus confronted with what may be a trivial issue but one that can alter the user's interpretation if an inappropriate classification is used. Each of these systems has its uses, given appropriate circumstances. For example equal intervals are useful for making comparisons between different maps of related data. However, 
as Cromley and Cromley (2009) note, equal interval classifications provide no indication of the statistical distribution of the underlying data; for this, classes based on standard deviations are suitable. Nevertheless, other classification schemes are also useful depending on what point is trying to be communicated in a map (see Indrayan and Kumar, 1996; Brewer, 2006).

A recent cartographic study of public health practitioners using interactive, e-health atlases (Koenig, 2008) has shown that novice health map users may need additional, textual support in interpreting classification systems. PHC informaticians need to consider the expertise level of map users when classifying, visualising and conveying geographic information: providing documentary support for users to aid cartographic communication is imperative. In our view, where there is doubt in classifying data, we recommend the use of quantiles, particularly quintiles, because of their common use in public health for displaying graphical information both in articles (eg, Christie et al., 2005; Adams and White, 2006) and in internet-based public health atlases.

\section{Map overlay}

GIS is essentially the integration of cartography, database technology and geographical analysis. One of its most fundamental facets is map overlay, which refers to the ability to superimpose maps (spatial database layers) of different phenomena upon each other in a GIS package to assess geographic co-location where relevant. A very basic example of map overlay would be superimposing general practitioner (GP) locations (a point layer) onto maps of socio-economic deprivation (area/polygon layers) to visualise the catchment context of a practice. A real-world case in point is the use of map overlay by Nottingham Primary Care Trust (PCT) to compare the geography of provision in New Leaf smoking cessation services (the first layer in the overlay) with that of high-priority target groups defined using geodemographics (the second layer in the overlay), enabling the identification of two highly localised patches of low provision but high needs (Packham and Robinson, 2009). For further reading on the application of geodemographics in PHC, readers are referred to Powell et al. (2007), which appeared in Primary Health Care Research

Primary Health Care Research \& Development 2012; 13: 10-21
\& Development. Using a map overlay for this purpose aided a resource allocation rethink such that within a year both patches had improved access to cessation services.

Many examples of the use of overlay techniques in epidemiological and health services analyses exist (eg, Luo, 2004; Wang and Luo, 2005). For instance, Field (2000) used a map overlay of indicators of population morbidity, socioeconomic status, transport access and built environment quality to produce an Index of Relative Disadvantage to identify localities with higher healthcare needs co-located with lower accessibility to GP clinics. Overlay operations typically involve bringing two layers together to form a new dataset made of some combination of the first two layers, for example to identify how many clinics (an example of a point layer) are within a census tract (termed a polygon layer in GIS terminology) so as to create a new dataset consisting only of the shared areas between the two original layers. Another common technique is the union operation, which enables the creation of a new layer consisting of all areas in the original two layers combined, such as when adjoining neighbouring census tracts to produce a larger area. Table 1 summarises some common overlay operations, their purposes and example PHC applications.

\section{Spatial analyses}

Beyond basic mapping, the use of GIS in PHC also extends to analytic purposes, which can vary from assessing access to services, to identifying patterns in clinical outcomes, to producing local estimates of population disease risk. In this section, relevant methods are critiqued in two subsections pertaining to two core concepts from the health services domain of the Faculty of Public Health, three domains of the public health paradigm (Griffiths et al., 2005), clinical effectiveness and services planning, respectively, as foundations for efficiency, audit and evaluation ultimately aiming towards equity.

\section{Analytic methods for investigating clinical effectiveness}

Detecting clusters and outliers are novel and growing aspects of spatial analysis in PHC. 
Table 1 Some common overlay operations, their purposes and example primary health care applications

\begin{tabular}{|c|c|c|}
\hline Overlay operation & Purpose & Example application \\
\hline Buffer & $\begin{array}{l}\text { To create a zone around a feature of a } \\
\text { specified distance. }\end{array}$ & $\begin{array}{l}\text { Determining residence within a distance-based } \\
\text { buffer around an industrial area considered } \\
\text { unsuitable for the development of healthcare } \\
\text { facilities. }\end{array}$ \\
\hline Clip & $\begin{array}{l}\text { To identify places from one GIS layer that lie } \\
\text { completely within the boundary of another } \\
\text { defined. }\end{array}$ & $\begin{array}{l}\text { Selecting GP catchment areas within the } \\
\text { catchment area of a specific hospital. }\end{array}$ \\
\hline Intersection & $\begin{array}{l}\text { To select shared areas from two layers so as } \\
\text { to create a new layer consisting only of the } \\
\text { shared areas. }\end{array}$ & $\begin{array}{l}\text { Identifying overlaps between the catchment } \\
\text { areas of two clinics. }\end{array}$ \\
\hline Point in polygon & $\begin{array}{l}\text { To determine whether a point location is } \\
\text { located inside or outside a polygon. }\end{array}$ & $\begin{array}{l}\text { Determining whether a patient's residence is } \\
\text { within an official catchment area. }\end{array}$ \\
\hline Union & $\begin{array}{l}\text { To create a new GIS layer consisting of all } \\
\text { areas in the original two layers combined. }\end{array}$ & $\begin{array}{l}\text { Joining two catchment areas to create a new } \\
\text { larger catchment area. }\end{array}$ \\
\hline $\begin{array}{l}\text { Map algebra } \\
\text { (raster overlay) }\end{array}$ & $\begin{array}{l}\text { A mathematical operation that can be } \\
\text { performed on one or more attributes for the } \\
\text { same cell that can be applied to all cells in } \\
\text { the overlay. The technique makes it possible } \\
\text { to overlay raster layers, creating a new layer } \\
\text { that is a combination of the original two } \\
\text { layers by performing an algebraic operation } \\
\text { on the two overlaying cells. }\end{array}$ & $\begin{array}{l}\text { By rating a catchment area for chronic disease } \\
\text { prevalence using a scale (1-5) at the individual } \\
\text { cell level, it makes it possible to add } \\
\text { overlapping cells and determine an overall } \\
\text { rating for each cell in relation to the prevalence } \\
\text { of chronic disease in each; the higher the rating, } \\
\text { the higher the prevalence of chronic diseases in } \\
\text { that cell. }\end{array}$ \\
\hline
\end{tabular}

GIS = geographic information systems; GP = general practitioner.

In relation to routine surveillance and regulation, it is in essence the ability to detect statistically significant spatial clusters and outliers in service performance, as well as disease (risk) hotspots. There are a range of different techniques to this end including Local Moran's I, Getis Ord Gi* statistic and Geary's C (Cromley and McLafferty, 2002: 130-57). The key concept to understand is that all of these measures are based on the comparison of the value of a given variable (eg, prevalence of coronary heart disease, CHD) at a specified location with values at proximate locations up to a specified threshold distance, in what is technically termed spatial autocorrelation. Thus, statistically significant clusters (hotspots) and outliers (coldspots) can be detected, and this ability should be distinguished from clusters suggested by eye gazing a map; subjective visual perceptions can sometimes suggest false clusters and outliers as commented on in the opening to the section on mapping health and health care. A primary care application of spatial cluster/outlier detection can be found in Nacul et al. (2011) in which the Local Moran's I test was used to compare observed PHC-based prevalence of chronic obstructive pulmonary disease with estimated prevalence based on epidemiological models for the purpose of identifying clusters of underdiagnosis for resource allocation purposes.

A caveat to the use of cluster/outlier detection techniques, however, is the existence of the modifiable areal unit problem, a prime but often neglected issue that refers to the effect that observed patterns can potentially change at different geographic scales (the scalar effect, a form of data aggregation/disaggregation effect) and with the use of different areal units (the zonal effect). However, it should be noted that Stafford et al. (2008) used self-reported health datasets on 1550 people at the individual postcode level, from the 1999 Health Survey for England, aggregated to three alternative small area configurations firstly census wards, secondly delineations according to built environment and thirdly units based on housing type - and found only a minor variation in the patterns observed between the three configurations. This suggests that the zonal effect might be negligible with respect to the type of routine health data used in PHC analyses. Nevertheless, the choice of spatial scale appears to be important as shown by comparison between the findings of Samarasundera et al. (2010b) and 
Soljak et al. (2011) regarding clustering of stroke under-diagnosis in primary care reveals. The Soljak et al. study examined patterns at the PCT level, finding evidence for significant clustering of under-diagnosis across Greater London using PCT-level data; however, parallel work by Samarasundera et al. (2010b) using practice-level datasets largely yielded significant clusters for practices in inner London, with few clusters remaining for outer London at the practice level. The lesson to learn from this and other studies on the same topic (eg, Schuurman et al., 2007; Callaghan et al., 2009) is that it is important to perform cluster/outlier detection at more than one scale to assess scalar effects.

However, cluster/outlier detection is not the only spatial analytic approach relevant to assessing clinical effectiveness. Spatial forms of regression, termed spatial regression, also have relevance. Traditional regression has a long history in healthrelated analyses, including in health geography, to evaluate health outcomes in relation to other variables such as socio-economic status. An illustrative example of the application of traditional regression analysis in a PHC context is Busato and Kunzi (2008). In the study, consultation rates were regressed against health care supply and sociodemographic factors in multivariate models for localities across Switzerland, enabling the researchers to assess the impacts of local contextual factors for utilisation behaviour.

While traditional regression is well suited for many analytical purposes, it is nevertheless nonspatial in structure and hence does not account for the effects of spatial proximity (eg, between adjacent census tracts) or boundary effects (eg, census tract boundaries are convenient but artificial, and thus rarely represent actual physical boundaries) as has been considered in the previous sub-section. As a result, those engaged in more complex analyses are increasingly using spatially explicit regression methods. While each method of spatial regression differs, there is a core similarity in that they all divide datasets into geographic sub-samples and perform local regression around each regression point (ie, data location) rather than on the full dataset. Subsamples are defined based on a statistical matrix based on the data in question, a process typically implemented in software packages automatically using spatial algorithms.
A spatial regression approach gaining increasing attention is the use of spatial lag and error models (Anselin, 1988), which although initially developed in the disciplines of spatial econometrics and economic geography, has been found to be useful in many other application areas including the health sector (eg, Emch et al., 2006; Mobley et al., 2006). A primary care application of this method is demonstrated by Mobley et al. (2006), who used spatial lag and error regression to identify localised variations in the effects of PHC factors and area context on hospital admissions for primary care sensitive conditions (PCSC) such as asthma and ear infections. Higher local rates in PCSC than the national average may indicate, to continue with the same disease examples, unmanaged asthma and inadequately treated ear infections. Indeed, one of the key findings of the study was that localities with lower levels of clinical support for GPs (eg, practice nurses) correlated strongly with higher admission rates. It is worth stressing that this correlation became more apparent in the Mobley et al. (2006) study with the use of spatial lag and error models than with classical regression models, and greatly aided the locality triangulation process. A point to consider nonetheless is that there is not always a spatial structure in regression relationships (technically termed spatial autocorrelation) and, under these circumstances, traditional regression will work just as effectively. While it may seem a daunting task to know when to use traditional regression and when to use spatial regression, the relevant software packages (see the sub-section on GIS and spatial statistics software for relevant websites) always include at least one test for spatial autocorrelation as a form of good practice.

\section{Analytic methods for services planning}

Perhaps the most obvious geographical aspect of PHC analysis is assessing spatial accessibility, which effectively is essential for service planning. Network analysis plays a fundamental role in this respect (eg, Wang and Luo, 2005) as it allows the travel distance from a particular postcode or the centre point of a census tract (centroid) to the nearest GP to be calculated using road, or other transport, networks and offers a level of sophistication beyond the more simplistic approach of using straight line (Euclidean) distances that 
many studies have used successfully (eg, Damiani et al., 2005; Propper et al., 2007). It is important to note that Euclidean distance does not always reflect the actual distances travelled to access health care using network methods and analysts should compare both measures whenever feasible as Jordan et al. (2004) have demonstrated.

Typical GIS software also allows the calculation of drive times based on speed limits for given stretches of roads. Furthermore, it is possible to select specific roads required in the analysis depending on the criteria, for example the Jordan et al. (2004) study incorporated primary, secondary and minor roads, given that these would typically be utilised when accessing a local GP clinic with roads such as motorways excluded. Their research concluded that drive time is a more accurate measure of access to health care especially in rural areas when compared with Euclidean distance. Network analysis can also be combined with spatial regression techniques for investigating accessibility and utilisation patterns, to explore how local variations in travel time vary in relation to localised pockets of deprivation in order to identify 'hotspots' of co-variation in poor spatial accessibility with less economically advantaged communities. Indeed, a growing number of studies have shown that doing so provides greater insights than traditional regression methods (Dawe, 2008; Bagheri et al., 2009; Jattansingh, 2009; Walsh, 2009).

A further analytic method relevant to service planning is kernel density smoothing, which is used to interpolate between point-based data (eg, practice locations) or to smooth census tract estimates of a phenomenon (eg, attendance rates) to account for boundary effects (smoothing is the re-estimation of the observed value at a tract based on not only the observed value but also its neighbours). Sociodemographic boundaries are by and large convenient abstractions rather than representing real-world boundaries. However, their use in analytical research can sometimes introduce problems. While for most routine monitoring purposes artificial boundaries are not a significant issue, the issue can sometimes become problematic when attempting to model processes and patterns, for example in healthcare utilisation behaviour. Smoothing techniques also have relevance to interpolating values between locations to provide estimates for places where data have not been collected or are unavailable.

One application of such a technique by Guagliardo (2004) used kernel density smoothing in order to investigate the spatial accessibility of primary care. Here, a continuous density layer was created from points representing physician accessibility across a city. The kernel density method made it possible to calculate the average physician density for each neighbourhood, with smoothing allowing for census tract border crossing to be accounted for. A similar method was adopted by McLafferty and Grady (2004; 2005), whereby prenatal care and access are analysed using kernel density estimation to establish the density of prenatal clinics in relation to mothers' residential locations. Figure 1 illustrates the use of kernel density smoothing to produce a continuous disease map using practice-level point pattern data in the form of Quality Outcomes Framework-based (Sigfrid et al., 2006; Department of Health, 2008: 9) decile-based prevalence estimates for CHD in northern England.

The limitation of kernel density approaches is that the shape of the kernel is inherently arbitrary and does not consider the spatial arrangement of the data in question, such that localities with a high density of points are smoothed in the same way as those with sparse points. Hence, more sophisticated interpolation methods such as geostatistics (commonly termed kriging) are sometimes appropriate, although the use of such techniques is beyond the scope of routine PHC mapping and analysis and are hence not critiqued here. For an introduction to geostatistics in health, readers are referred to Cromley and McLafferty (2002: 200-204); advanced readers are referred to Webster and Oliver (2007).

\section{Software, the internet and organisational issues}

There are a range of packages available for mapping and analyses to implement the methods discussed thus far in this review, with different software types having different purposes and different specific packages having differing strengths and weaknesses. In addition to these desktop programs, there are also a range of internet-based sites offering mapping and sometimes analytic functionality. Internet-based GIS facilities offer no- or lowcost solutions requiring little or no domain expertise for basic health surveillance and services planning; more complex analytic work of the type previously discussed with respect to tasks such as identifying spatial variation in under-diagnosis and interpolating 


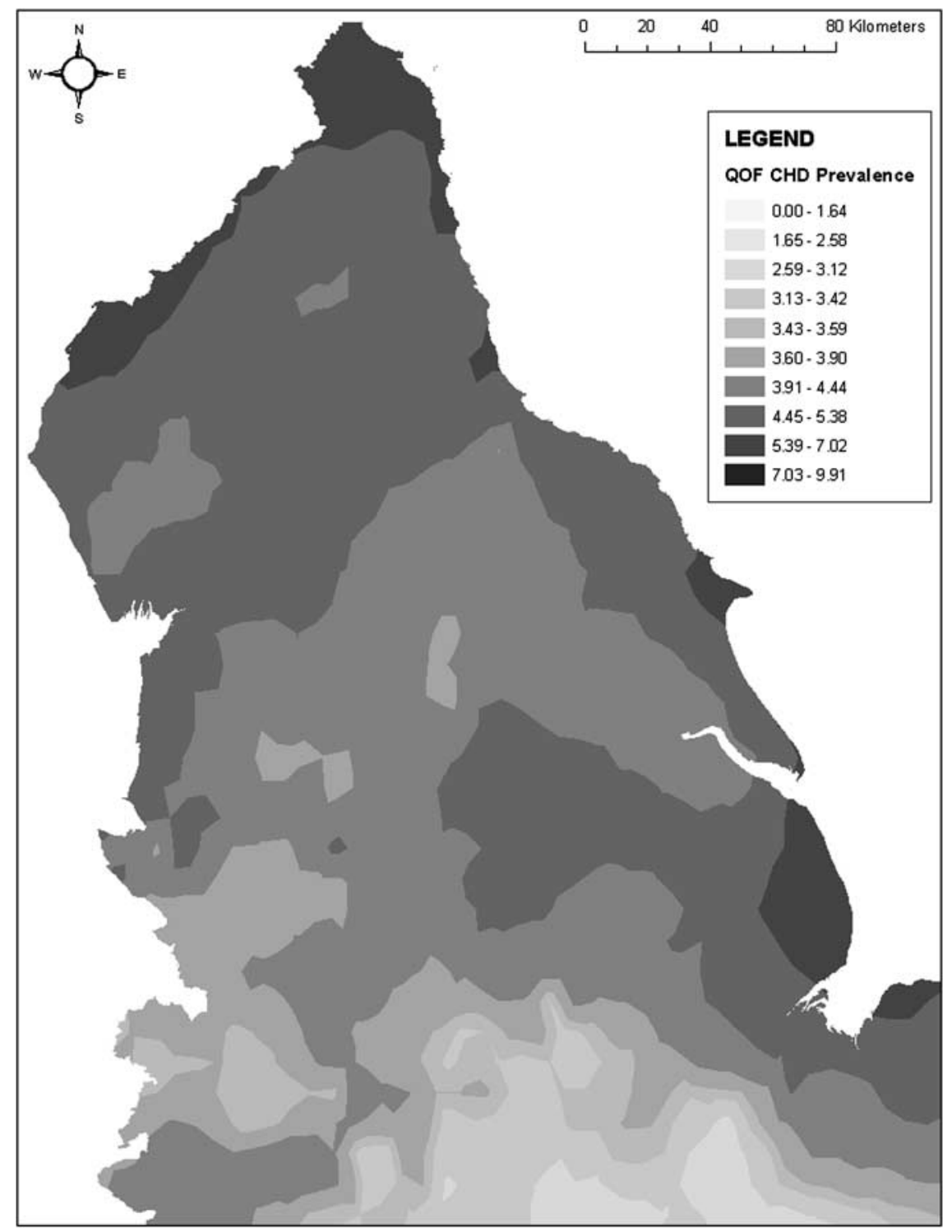

Figure 1 Predicted coronary heart disease prevalence based on an inverse-squared distance kernel density model

clinic-level prevalence datasets requires domain knowledge and specialist software. Selecting the fit-for-purpose tools and the associated institutional considerations are discussed in this section.

\section{Internet-based mapping}

With respect to core mapping of local indicators, the growth of interactive, e-health atlases

Primary Health Care Research \& Development 2012; 13: 10-21 underpinned by GIS software has provided health intelligence with valuable routine locality profiling tools. Many of these atlases and tools are operated by public health bodies, for example the New Zealand Ministry of Health Public Health Intelligence (http://www.phionline.moh.govt.nz/) and the North West Public Health Observatory Health GIS (http://www.nwpho.org.uk/healthgis/). Such atlases are typically produced using cut-down 
GIS packages such as InstantAtlas (http://www. instantatlas.com/), which require little domain expertise other than familiarity with spreadsheets, and are thus readily deployable in PHC settings without significant additional staff training, outsourcing or buying in of new expertise. The functionality provided by such packages invariably enables users to link to graphical data such as line graphs on time trends in disease for a given area or funnel plots for locating performance outliers.

Some of these public health atlases include datasets specific to PHC, a case in point being the New Zealand Ministry of Health Public Health Intelligence interactive atlas (http://www.phionline. moh.govt.nz/), which includes a primary care section summarising data on PHC-based prevalence, utilisation and unmet needs. The facility allows users, for example, to identify unmet needs in a primary care organisation by clicking on its name in the accompanying table. Doing so then highlights that organisation on the map and on the accompanying graph so that comparisons can be made with neighbouring bodies.

\section{Value-added tools and customised services}

Similar mapping functionality is available for many internet- and network-based tools and services such as the National Health Service (NHS) Strategic Health Asset Planning and Evaluation (SHAPE) mapping facility in the United Kingdom (http://shape.dh.gov.uk/public/mapping.asp), HealthLandscape Primary Care Atlas in the United States of America (http://www.health landscape.org/) and iQ HealthMaps in the United Kingdom (http://www.iq-medical.co.uk/productshealthmaps.php). Such tools are typically customised, internet-enabled GIS software that offer not only basic mapping facilities but also some spatial analytic functionality and often include useful datasets within them; hence, they can be considered as value-added tools. For example iQ HealthMaps integrates Google-based mapping with the ability to perform basic point pattern mapping of patient residences and also allows the overlay of integrated datasets such as MOSAIC geodemographic classifications (see Webber, 2004 for a discussion on the use of MOSAIC in health services analysis), aspects of the built environment and healthcare infrastructure without the need for specialist skills on the part of the user.
Both SHAPE and iQ HealthMaps offer users the ability to explore scenarios such as broad-level impacts of the closure and opening of new healthcare facilities upon communities by overlay different GIS layers such as morbidity rates, drive-time distances and sociodemographic data. While broadly similar in facilities, nevertheless, some clear distinctions can exist even between value-added tools. For example among the examples already cited, SHAPE is state-run and free to NHS staff whereas iQ HealthMaps is a commercial product; HealthLandscape Primary Care Atlas and its associated tools are free to a certain extent but are at a cost for their more sophisticated facilities.

The examples discussed above are but some of a range of tailored solutions available, Managers of PHC information should carefully consider whether it is cartographic visualisation (eg, mapping disease trends) or spatial analysis (eg, calculating drive times for accessibility analysis) that is more relevant from their organisational perspective, and exactly what functionality they require in order to successfully co-ordinate the use of different GIS solutions products as part of fit-forpurpose healthcare intelligence systems.

\section{GIS and spatial statistics software}

For those involved (or wishing to develop capacity) in spatial analytic work such as statistically based cluster/outlier detection, complex desktopbased packages are a fundamental addition to the range of tools already discussed. As GIS continues to penetrate healthcare research and development, it is likely that spatial analysis will be the next geography-related trend in primary care informatics to complement and build on the current proliferation of healthcare mapping, although currently full analytical packages are largely used by epidemiologists and many organisations in the public health sector (eg, health protection bodies) rather than by those focusing on PHC. This is especially likely in countries such as the United Kingdom, where Joint Needs Assessment collaborations with local governmental authorities linked to urban renewal (Blackman, 2006; National Health Service London Healthy Urban Development Unit, 2006; Land Use Consultants, 2007) require integrated, medium-term spatial planning.

There is a range of relevant software for analytical purposes including mainstream GIS software 
such as ESRI ArcGIS (http://www.esri.com/software/arcgis/index.html) and MapInfo (http:// www.pbinsight.eu/uk/products/location-intelligence/), which also provide mapping and spatial database management facilities, as well as more specialist spatial statistics packages such as GeoDa (http:// geodacenter.asu.edu/geodasum) and GWR (http:// ncg.nuim.ie/ncg/GWR/software.htm). PHC informaticians, managers and planners working at highly local community levels are unlikely to need such software, at least in the context of the majority of More Economically Developed Countries (MEDCs), considering the range of tailored software and internet mapping presently available in such parts of the world. However, PHC researchers and developers working with larger datasets and with greater analytic needs, such as those planning at the strategic level, should seriously consider deploying such tools if this has not been done already. In the second main section of this review, the use of cluster/ detection techniques, spatial regression and interpolation was discussed in relation to primary care, but what about deciding whether to actually deploy the relevant software within a given organisation? A not uncommon mistake, across many employment sectors, is to introduce complex geographical software without (a) an overall strategy and goals in mind and (b) without appropriately trained personnel. Also, the appropriate use of statistical techniques always requires domain knowledge and should be viewed as a distinct activity in itself. A GIS package is suitable when an organisation requires significant in-house management of spatial data as well as the abilities to perform cartographic and analytic work. Spatial statistics packages can often be a valuable foil to the use of GIS software for such purposes, especially in contexts where visual identification of clusters/outliers is insufficient, thus requiring more formal methods of detection and for whom statistical triangulation of PHC performance indicators is the core activity.

Nevertheless, relatively recent studies of the use of such tools in healthcare organisations in MEDCs reveal much room for further productive uptake of spatial analytic tools. For example Smith et al. (2003) and Higgs et al. (2005) researched GIS use in the British NHS, and while the studies showed increasing awareness of the benefits of such tools, no doubt a contributing factor to the present usage levels of internet-based mapping and value-added tools, a key obstacle was found to be limited data sharing between healthcare bodies. This hurdle was found to be more evident regarding data sharing between the healthcare sector and local authorities, a source for concern considering the importance of collaborative health improvement strategies. With respect to the types of PHCrelevant spatial analysis being carried out in the NHS, the Smith et al. study was revealing in that much of the analytic uses of GIS in 2001 are little different from those we can observe today, the most common applications being for monitoring locality healthcare needs and planning catchment areas. There was little evidence of the use of GIS for modelling purposes and forecasting, a shortfall that is gradually changing, although it is the private sector that has successfully come to dominate this growth niche and aid in taking this invaluable agenda forward in the United Kingdom (eg, Dr Foster Population Health Manager: http://www. drfosterintelligence.co.uk/managementInformation/ $\mathrm{PHM} /$ ).

An interesting strategic solution to the organisational implementation of spatial analysis is that adopted in New Zealand, where the Ministry of Health has developed a joint initiative with the University of Canterbury to set up the GeoHealth Laboratory, which provides ongoing geographical intelligence for both disease monitoring and care development (http://www.geohealth.canterbury.ac. nz/; Pearce, 2007); some of the laboratory's work directly relates to primary care (eg, Hiscock et al., 2008) although the laboratory's remit extends to the wider health services infrastructure as well as to epidemiological studies.

The situation is somewhat complicated in Lesser Economically Developed Countries, with limited availability of the relevant tools through PHC in the 'Third World', which frequently overlaps with health protection issues relating to infectious disease epidemiology anyway, can follow the lead of environmental health specialists by making use of Google Maps and Google Earth for basic mapping (eg, Lozano-Fuentes et al., 2008) and combining their use with some of the freeware analytic packages available such as GRASS and GeoDa. Interfacing by the PHC sector in such countries with non-governmental organisations and the WHO with respect to developing GIS capacity could be a potentially invaluable contribution to the meeting of stated WHO goals in programmes such as the African Healthy Cities 
(World Health Organization, 2003) and Closing the Gap in a Generation (World Health Organization, 2008) programmes.

\section{Summary and future directions}

The mainstreaming of spatial mapping and analysis techniques and toolkits has provided the means by which to utilise a growing range of routinely available geographic data sources for PHC research and development. However, careful consideration of resource issues and strategic goals is paramount for successful implementation within an organisation.

Developing collaborative networks within the health sector as well as with academic institutions can greatly aid this agenda, as evidenced by the role of the Association for Geographic Information (AGI) Local Public Services Special Interest Group (http://www.agi.org.uk/lps-welcome). Data sharing and interdisciplinary work would be essential for any such networks to be fruitful, topics previously raised in environmental health circles as being important to progress GIS use further in that area of health (Dunn et al., 2007). The biggest obstacle to this goal appears to be a lack of awareness of the potential of GIS for analysis and modelling, and even the existence of spatial statistics, within health services organisations including at senior levels (Smith et al., 2003; Higgs et al., 2005; Joyce, 2009), and this may be the first thing that needs to be addressed. Organisations such as the United Kingdom's AGI and its equivalents in other countries will be pivotal to the growth of primary care GIS.

With the topic of developing the geographical mapping and analysis agenda in mind, what aspects of this agenda need further research and development? Certainly GIS is well established within epidemiology, health services and public health at least to the extent that there are standard textbooks on the subject (eg, Cromley and McLafferty, 2002; Craglia and Maheswaran, 2004; Skinner, 2010) but what of the specific case of primary care? Starting from the literature base, while there are certainly many public health journals that publish geographically related articles, as well as two journals specialising in health geography (Health and Place, International Journal of Health Geographics), a search for the terms 'primary health care' or 'primary care' in co-occurrence with GIS-related terms reveals a dearth of literature pertaining to statistical, internet-based tools explicitly for PHC development. This literature gap mirrors the tools gap, with the exception of Dr Foster Population Health Manager, which, in many respects, is moving in this direction.

Nevertheless, there are areas in which synergy between PHC bodies, public health intelligence and academia could develop internet-based tools for the routine use of statistical cluster/outlier detection and spatial regression. While actual processing tools for such techniques would be too computationally intensive for current internet technology, a useful step forward would be the development of interactive atlases that provide only the results of spatial analysis pertaining to primary care data. A hypothetical example of this could be an interactive atlas of clusters/outliers in modelled under-diagnosis in PHC across a range of chronic conditions, utilising the results of analytic studies such as Nacul et al. (2011). Such a tool could aid practices and regulatory bodies in identifying disease priorities for service development and compare performance with neighbouring institutions.

Geographical mapping and analysis aimed specifically at PHC is still at an early stage. To demonstrate its potential for cutting-edge development to those working in the 'front line' of informing and managing service delivery, collaboration between academics working, respectively, in GIS and PHC to produce proof-of-concept demonstrator systems for analytic, internet-based PHC tools might be the best starting point.

\section{References}

Adams, J. and White, M. 2006: Removing the health domain from the Index of Multiple Deprivation 2004 - effect on measured inequalities in census measure of health. Journal of Public Health 28, 379-83.

Anselin, L. 1988: Spatial econometrics: methods and models. Dordrecht: Kluwer Academic Publishers.

Bagheri, N., Holt, A. and Benwell, G.L. 2009: Using geographically weighted regression to validate approaches for modelling accessibility to primary health care. Applied Spatial Analysis 2, 177-94.

Blackman, T. 2006: Placing health: neighbourhood renewal, health improvement and complexity. Bristol: Policy Press.

Brewer, C.A. 2006: Basic mapping principles for visualizing cancer data using geographic information systems. American Journal of Preventive Medicine 30, S25-36. 
Brewer, C.A. and Pickle, L. 2002: Evaluation of methods for classifying epidemiological data on choropleth maps in series. Annals of the Association of American Geographers 92, 662-81.

Brewer, C.A., MacEachren, A.M., Pickle, L.W. and Herrmann, D. 1997: Mapping mortality - evaluating colour schemes for choropleth maps. Annals of the Association of American Geographers 87, 411-38.

Busato, A. and Kunzi, B. 2008: Primary care physician supply and other key determinants of health care utilisation: the case of Switzerland. BMC Health Services Research 8, 8.

Callaghan, M., Cormican, M., Prendergast, M., Pelly, H., Cloughley, R., Hanahoe, B. and O'Donovan, D. 2009: Temporal and spatial distribution of human cryptosporidiosis in the west of Ireland 2004-2007. International Journal of Health Geographics 8, 64.

Christie, S., Morgan, G., Heaven, M., Sandifer, Q. and van Woerden, H. 2005: Analysis of renal service provision in south and mid Wales. Public Health 119, 738-42.

Cossman, R.E., Cossman, J.S., Jackson, R. and Cosby, A. 2003: Mapping high or low mortality places across time in the United States: a research note on a health visualisation and analysis project. Health and Place 9, 361-69.

Craglia, M. and Maheswaran, R. 2004: GIS in public health practice: opportunities and pitfalls. Boca Raton, FL: CRC Press.

Cromley, R.G. and Cromley, E.K. 2009: Choropleth map legend design for visualizing community health disparities. International Health Geographics 8, 52.

Cromley, E.K. and McLafferty, S.L. 2002: GIS and public health. New York: Guilford Press.

Crooks, V.A. and Andrews, G.J. 2009: Community equity access: core geographic concepts in primary health care. Primary Health Care Research \& Development 10, 270-73.

Damiani, M., Propper, C. and Dixon, J. 2005: Mapping choice in the NHS: cross-sectional study of routinely collected data. British Medical Journal 330, 284-89.

Dawe, A. 2008: Network analysis of road access to $A \& E$ departments in a metropolitan area. London: Unpublished Master of Science dissertation thesis, University College London.

Department of Health. 2008: Developing the quality and outcomes framework: proposals for new, independent process. London: Department of Health.

Dunn, C.E., Bhopal, R.S., Cockings, S., Walker, D., Rowlingson, B. and Diggle, P. 2007: Advancing insights into methods for studying environment-health relationships: a multi-disciplinary approach to understanding Legionnaires' disease. Health and Place 13, 677-90.

Emch, M., Ali, M., Park, J.-K., Yunus, M., Sack, D.A. and Clemens, J.D. 2006: Relationship between neighbourhoodlevel killed oral cholera vaccine coverage and protective efficacy: evidence for herd immunity. International Journal of Epidemiology 35, 1044-50.

Field, K. 2000: Measuring the need for primary health care: an index of relative disadvantage. Applied Geography 20, 305-32.

Primary Health Care Research \& Development 2012; 13: 10-21
Griffiths, S., Jewell, T. and Donnelly, P. 2005: Public health in practice: the three domains of public health. Public Health 119, 907-13.

Guagliardo, M.F. 2004: Spatial accessibility of primary care: concepts, methods and challenges. International Journal of Health Geographics 3, 1-13.

Higgs, G., Smith, D.P. and Gould, M.I. 2005: Findings from a survey on GIS use in the UK National Health Service: organisational challenges and opportunities. Health Policy 72, 105-17.

Hiscock, R., Pearce, J., Blakely, T. and Witten, K. 2008: Is neighbourhood access to health care provision associated with individual-level utilisation and satisfaction? Health Services Research 43, 2183-200.

Indrayan, A. and Kumar, R. 1996: Statistical choropleth cartography in epidemiology. International Journal of Epidemiology 25, 181-89.

Jattansingh, K. 2009: Determining differences in access to accident and emergency departments using 2SFCA and GWR. London: Unpublished Master of Science dissertation thesis, University College London.

Jordan, H., Roderick, P., Martin, D. and Barnett, S. 2004: Distance, rurality and the need for care: access to health services in South West England. International Journal of Health Geographics 3, 21.

Joseph, A.E. and Phillips, D.R. 1984: Accessibility and utilization: geographical perspectives on health care delivery. London: Harper and Row.

Joyce, K.E. 2009: Public health decision-makers' perceptions of the value of geographical information systems (GIS). Health and Place 15, 831-40.

Koenig, A. 2008: Visualisation of population morbidity patterns in health care decision-making. London: Unpublished Master of Science dissertation thesis, University College London.

Land Use Consultants in association with the Centre for Research into Environment and Health. 2007: Delivering healthier communities in London. London: HUDU.

Lawson, A.B. 2001: Tutorial in biostatistics: disease map construction. Statistics in Medicine 20, 2183-204.

Lawson, A.B. and Williams, F.L.R. 2001: An introductory guide to disease mapping. London: Wiley.

Lozano-Fuentes, S., Elizonda-Quiroga, D., Farfana-Ale, J.A., Lorono-Pino, M.A., Garcia-Rejon, J., Gomez-Carro, S., Lira-Zumbardo, V., Najera-Vazquez, R., Fernandez-Salas, I., Calderon-Martinez, J., Dominguez-Galera, M., Mis-Avila, P., Morris, N., Coleman, M., Moore, C.G., Beaty, B.J. and Eisen, L. 2008: Use of Google Earth ${ }^{\mathrm{TM}}$ to strengthen public health capacity and facilitate management of vector-borne diseases in resource-poor environments. Bulletin of the World Health Organization 86, 718-25.

Luo, W. 2004: Using a GIS-based floating catchment method to assess areas with shortage of physicians. Health and Place 10, 1-11.

Mackie, P. and Sim, F. 2008: Here be dragons. Public Health 122, 737-38. 
McLafferty, S. and Grady, S. 2004: Prenatal care need and access: a GIS analysis. Journal of Medical Systems 28, 321-33.

McLafferty, S. and Grady, S. 2005: Immigration and geographic access to prenatal clinics in Brooklyn, NY: a geographic information systems analysis. American Journal of Public Health 95, 638-40.

Meade, M.S. and Earickson, R.J. 2005: Medical geography, Ch. 11. New York: Guilford Press, 381-400.

Mobley, L.R., Root, E., Anselin, L., Lozano-Gracia, N. and Koschinsky, J. 2006: Spatial analysis of elderly access to primary care services. International Journal of Health Geographics 5, 19-35.

Nacul, L., Soljak, M., Samarasundera, E., Hopkinson, N.S., Lacerda, E., Indulkar, T., Flowers, J., Walford, H. and Majeed, A. 2011: COPD in England - a comparison, model-based prevalence and observed prevalence from general practice data. Journal of Public Health 33, 108-16.

National Health Service London Healthy Urban Development Unit. 2006: London Thames Gateway Social Infrastructure Framework: a toolkit to guide decision making. London: HUDU.

Packham, C. and Robinson, J. 2009: Practical uses of GIS in a Primary Care Trust. Nottingham: Nottingham City NHS.

Pearce, J. 2007: Incorporating geographies of health into public policy debates: the GeoHealth Laboratory. New Zealand Geographer 63, 149-53.

Powell, J., Tapp, A., Orme, J. and Farr, M. 2007: Primary care professionals and social marketing of health in neighbourhoods: a case study approach to identify target and communicate with 'at risk' populations. Primary Health Care Research \& Development 8, 22-35.

Propper, C., Damiani, M., Leckie, G. and Dixon, J. 2007: Impacts of patients' socio-economic status on the distance travelled for hospital admission in the English National Health Service. Journal of Health Services Research \& Policy 12, 153-59.

Ricketts, T.C. and Holmes, G.M. 2007: Mortality and physician supply: does region hold the key to the paradox? Health Services Research 42, 2233-51.

Samarasundera, E., Martin, D., Saxena, S. and Majeed, A. 2010a: Socio-demographic data sources for monitoring locality health profiles and geographical planning of primary health care in the United Kingdom. Primary Health Care Research \& Development 11, 287-300.

Samarasundera, E., Soljak, M., Indulkar, T. and Calderon, A. 2010b: Exploring the use of mapping and spatial statistics in the context of health and social care regulation. London: Imperial College London [Report for the Care Quality Commission].

Schuurman, N., Bell, N., Dunn, J.R. and Oliver, L. 2007: Deprivation indices, population health and geography: an evaluation of the spatial effectiveness of indices at multiple scales. Journal of Urban Health 84, 591-603.

Sigfrid, L.A., Turner, C., Crook, D. and Ray, S. 2006: Using the UK primary care Quality and Outcomes Framework to audit health care equity: preliminary data in diabetes management. Journal of Public Health 28, 221-25.

Skinner, R. 2010: GIS in hospital and healthcare emergency management. Boca Raton, FL: CRC Press.

Smith, D.P., Gould, M.I. and Higgs, G. 2003: Re(surveying) the uses of geographical information systems in Health Authorities 1991-2001. Area 35, 74-83.

Soljak, M., Samarasundera, E., Indulkar, T., Walford, H. and Majeed, A. 2011: Variations in cardiovascular disease under-diagnosis in England: cross-sectional spatial analysis. BMC Cardiovascular Disorders 11, 12.

Stafford, M., Duke-Williams, O. and Shelton, N. 2008: Small area inequalities in health: are we underestimating them? Social Science and Medicine 67, 891-99.

Walsh, T. 2009: Modelling geographic variation in primary health care provision. London: Unpublished Master of Science dissertation thesis, University College London.

Walter, S.D. 1992: The analysis of regional patterns in health data: I. Distributional considerations. American Journal of Epidemiology 136, 730-41.

Walter, S.D. and Birnie, S.E. 1991: Mapping mortality and morbidity patterns. International Journal of Epidemiology 20, 678-89.

Wang, F. and Luo, W. 2005: Assessing spatial and nonspatial factors for healthcare access: towards an integrated approach to identifying professional shortage areas. Health and Place 11, 131-46.

Webber, R. 2004: Neighbourhood inequalities in the patterns of hospital admissions and their application to the targeting of health promotion campaigns. London: CASA, University College London.

Webster, R. and Oliver, M.A. 2007: Geostatistics for environmental scientists. Chichester: Wiley.

World Health Organization. 2003: Healthy cities around the world. Copenhagen: WHO Regional Office for Europe.

World Health Organization. 2008: Closing the gap in a generation: health equity through action on the social determinants of health. Geneva: WHO. 